\title{
Primary Care, Behavioral Health, Provider Colocation, and Rurality
}

\author{
Benjamin F. Miller, PsyD, Stephen Petterson, PhD, Shandra M. Brown Levey, PhD, \\ Jessica C. Payne-Murphy, MA, Miranda Moore, PhD, \\ and Andrew Bazemore, $M D, M P H$
}

Purpose: The purpose of this study was to characterize the proximity of primary care and behavioral health service delivery sites in the United States and factors influencing their colocation.

Methods: We geocoded the practice addresses of primary care and behavioral health providers found in the Centers for Medicare \& Medicaid Services' National Plan and Provider Enumeration System Downloadable File to report where colocation is occurring throughout the country.

Results: The extent to which primary care physicians are colocated with behavioral health providers is strongly associated with rurality. Specifically, $\mathbf{4 0 . 2 \%}$ of primary care physicians in urban areas are colocated with behavioral health providers compared with $22.8 \%$ in isolated rural areas and $26.5 \%$ in frontier areas. However, when controlling for number of primary care physicians at a location, the odds of colocation actually are greater for physicians in a frontier area than those in urban areas (odds ratio, 1.289; $P<$.01).

Conclusions: Our findings offer new insights into the overlap of the behavioral health and primary care workforce, where opportunities for integration may be limited because of practice size and the proximity of providers, and where new possibilities for integration exist. (J Am Board Fam Med 2014; 27:367-374.)

Keywords: Health Policy, Integrated Delivery Systems, Mental Health, Primary Health Care

Current fragmentation in health care leads to increased cost and is associated with decreased health outcomes and overall quality of care. ${ }^{1-3}$ Central to this problem is the perpetuation of the false dichotomy that has separated mental health from physical health systems. ${ }^{4}$ Many have written extensively about the need for increased and improved integration of primary care and behavioral health, which includes mental health and substance use services. $^{4-7}$ Emerging research indicates that the

This article was externally peer reviewed.

Submitted 20 September 2013; revised 5 February 2014; accepted 10 February 2014.

From the Department of Family Medicine, University of Colorado Denver School of Medicine, Aurora (BFM, SMBL, JCP-M); and The Robert Graham Center, Washington, DC (SP, MM, AB).

Funding: none.

Conflict of interest: none declared.

Corresponding author: Benjamin F. Miller, PsyD, University of Colorado Denver School of Medicine Department of Family Medicine, Mail Stop F49, Academic Office 1, 12631 East 17th Avenue, Aurora, CO 80045 (E-mail: benjamin.miller@ucdenver.edu). integration of behavioral health into primary care improves health care access, minimizes stigma associated with seeking mental health services, increases overall health outcomes, and lowers health care costs. ${ }^{6,8}$

To date, there have been limited attempts to assess where integration is occurring throughout the country. ${ }^{9}$ The challenge for this assessment is the vast differences in practices' classification schemas for integration and the lack of a national database that clearly encapsulates the practices that are integrating primary care and behavioral health on site..$^{10,11}$

This article uses a novel approach to identify where behavioral health and primary care service delivery currently intersect and diverge, focusing on their proximity or colocation to each other. While colocation is by no means the equivalent of integration, it is often a part of the definition. ${ }^{10}$ Using practice addresses from a listing of most health care providers in the United States, we examined the extent to which primary care physicians (PCPs) and 
behavioral health providers share the same space, although not necessarily the same practice.

A somewhat self-evident issue is that colocation is more common in larger practices. What is not so obvious is the extent to which PCPs in smaller practices share space with behavioral health providers. Thus, we examine how the concentration of primary care providers in a particular location is related to colocation. A shared location (also known as colocation) also means something different in rural than urban areas. In particular, sharing the same address may be meaningful in an urban area, but colocation of rural PCPs and behavioral health providers in the same small town may matter the most. Sharing the same space, however defined, is certainly different from integration. Still, colocation is of intrinsic interest because it offers the potential of collaboration and the improvement of health care delivery for Americans.

Using US national workforce data, our analysis addresses 3 related issues. The first is a straightforward examination of the association between rurality and the number of PCPs that are colocated (hereafter "PCPs at location"), regardless of whether they are in the same practice. The second issue is the relationship between PCPs at location and colocation between primary care physicians and behavioral health providers, using both a narrow and broad measure of colocation. The final issue is less obvious: the extent to which the association between rurality and PCPs at location explains differences across levels of rurality in the colocation of PCPs and behavioral health providers.

\section{Methods}

The Health Insurance Portability and Accountability Act of 1996 (HIPAA) mandated that the National Provider Identifier (NPI) be required for Medicare services and has been widely adopted by other payers. ${ }^{12}$ In particular, in 2007, all entities covered by the Health Insurance Portability and Accountability Act, such as providers completing electronic transactions, health care clearinghouses, and large health plans, were required to use only the NPI to identify covered health care providers. Thus, nearly all health care providers who bill third parties for their services, including physicians and behavioral health providers, have obtained an NPI. Providers who are excluded include those who (1) only bill their patients directly or (2) occupy a salaried position and payment for their services is only from their employer. Information gathered from health care providers with an NPI is publicly available from the Centers for Medicare \& Medicaid Services in the National Plan and Provider Enumeration System (NPPES) Downloadable File. The data includes 2 pieces of provider information required for our colocation analysis: practice address and provider specialty. We used data from August 2010; this earlier date was chosen to minimize the inclusion of an increasing number of retired or otherwise inactive providers listed in the NPPES.

We were able to identify active physicians with more precision by matching the NPPES data with the 2010 American Medical Association's (AMA) Physician Masterfile. Using common identifiers in both data sets (including name, address, and unique physician identification number), we were able to match approximately $95 \%$ of the physicians in the 2010 NPPES data with those in the 2010 AMA Physician Masterfile. Given this match, we restricted our analysis to physicians classified in the Masterfile as providing direct patient care, thus excluding those who are retired residents, as well as those who mainly teach or hold administrative positions.

By geocoding the practice addresses in the NPPES data we were able to count the number of behavioral health providers and PCPs in a particular location. PCPs are those specializing in family medicine, general internal medicine, general pediatrics, geriatrics, and general practice. Behavioral health providers include psychiatrists, psychologists, social workers, marriage and family therapists, mental health counselors, and substance abuse counselors.

Using longitude and latitude coordinates, we used different levels of precision to identify a shared space. Using 5 digits after the decimal place (eg, 43.23983, 23.56778), there is approximately a $1-\mathrm{m}$ buffer around each point; this level of precision almost always means that persons at that location share the same street address. This is different from being in the same practice. It is quite common, especially in larger urban centers, that multiple medical practices share the same street address. At the same time, it is not uncommon for physicians and behavioral health providers in different practices to collaborate in the care of a pa- 
tient; such collaboration is arguably facilitated by proximity.

Using 4 digits (eg, 43.2398, 23.5678) there is approximately a $10-\mathrm{m}$ buffer that may include neighboring buildings; using 3 digits, the buffer is approximately $100 \mathrm{~m}$ ("down the road"); and using 2 digits, the buffer is $1 \mathrm{~km}$ (the "other side of a small town"). For each of these levels of precision we obtained a count of the behavioral health providers and PCPs and calculated the percentage of these physicians who are colocated with these providers. Our preliminary findings indicated little difference in estimates of colocation using 5, 4, or 3 digits; thus we restricted the analysis to results using the more precise (5-digit) measure and the broader 2-digit measure.

For each PCP, we obtained a count of the number of PCPs sharing the same 5-digit latitude and longitude, which is effectively the same street address (but not the same suite, office, floor, and any other ways to differentiate among multiple occupants that share the same street address.) We use "PCPs at location" as a shorthand for this measure, with understanding that multiple practices may be located at a particular site and that a practice may have providers located at multiple sites. For ease of presentation, we classified site size into 3 categories: just $1 \mathrm{PCP}, 2$ to 10 physicians, and $>10$ physicians.

To identify different levels of rurality, we use a measure based on the Rural-Urban Commuting Area (RUCA) codes. This is a Census tract-based classification scheme that uses the standard Bureau of Census Urbanized Area and Urban Cluster definitions in combination with work commuting information to characterize all the nation's Census tracts regarding their rural and urban status and relationships. There is also a ZIP code RUCA approximation that is used in this analysis by using the ZIP code of providers' practice location. Rural researchers have developed a RUCA-based measure that classifies all ZIP code areas as urban, large rural, small rural, isolated rural, or frontier. ${ }^{13}$ Among several alternatives, we use the following definition of frontier areas based on travel time: "all ZIPs that have RUCA 10 codes that are more than 60 minutes or greater road travel to the closest edge of an Urbanized Area and are more than 30 minutes or greater road travel to the closest edge of a large Urbanized Cluster of 10,000 population or greater." 13
We used simple tabulations and $\chi^{2}$ statistics to examine the associations between (1) PCPs at location and rurality, (2) PCPs at location and colocation, and (3) rurality and colocation. To examine the extent to which rural-urban differences are attributable to smaller numbers of PCPs at location in rural areas, we estimated multiple logistic regression models. Using both a precise (5-digit) and broad (2-digit) measure of colocation, we examined nested models. These include levels of rurality (urban, large rural, small rural, isolated rural, and frontier), PCPs at location, as well as an interaction term between PCPs at location and rurality. We compared different ways to specify PCPs at location and decided to use the natural log of size to capture the nonlinearity in the relation between this number and colocation (see Results). All statistical analyses were completed using Stata 13.1 (StataCorp LP, College Station, TX). Statistical significance is defined as $P<.01$.

\section{Results}

The first step of this analysis was to examine the relationship between level of rurality and the number of PCPs located at a particular location (PCPs at location). Overall, in the 2010 NPPES, we identified approximately 211,000 primary care physicians in direct patient care, a figure very close to other estimates of the size of the primary care workforce. ${ }^{14-16}$ Of these PCPs, 175,197 practice in urban areas, 18,113 in large rural areas, 9,837 in small rural areas, 2,560 in isolated rural areas, and 2,248 in frontier areas. As expected, there is a strong association between numbers of PCPs at location and rurality (Figure 1). The percentage of all locations with just 1 PCP is $12 \%$ in urban areas and doubles to $24 \%$ in isolated rural areas and $21 \%$ in frontier areas. The percentage of locations with more than 10 PCPs is $39 \%$ in urban areas but just $9 \%$ in frontier areas.

The extent to which PCPs are colocated with behavioral health providers is strongly associated with rurality using either a point (with 5 digits after the decimal) or an area with a $1.1-\mathrm{km}$ buffer (2 digits) (Figure 2). With the point estimate, we found that $40.2 \%$ of PCPs in urban areas are colocated with behavioral health providers compared with $22.8 \%$ in isolated rural areas and $26.5 \%$ in frontier areas. Using the larger area to define colocation, more than three fourths $(77.8 \%)$ of urban PCPs are near a behavioral 
Figure 1. Distribution of primary care physicians (PCPs) at location by level of rurality.

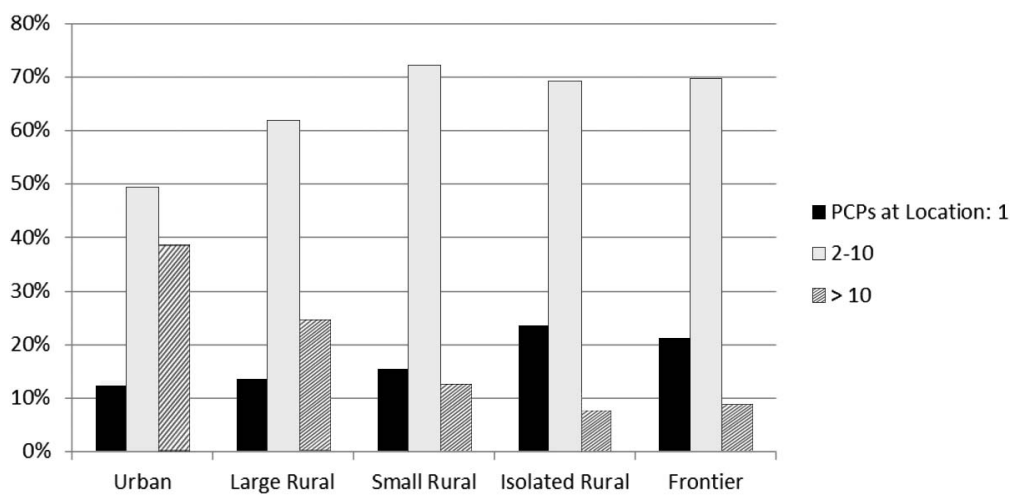

health provider compared with a little more than a third $(37.3 \%)$ in frontier areas.

We turn to the final analysis that examines differences in colocation across both rurality and numbers of PCPs at location. While there is a substantial difference in colocation across levels of rurality, we examined the extent to which these differences are explained by fewer PCPs at a given location. The nested logistic regression results in Table 1 summarize our analysis.

Our main finding is that after controlling for the number of PCPs at location, much of the observed difference in colocation across levels of rurality disappears. In particular, in the second model, there is not a significant difference in the likelihood of colocation in an urban area and an isolated rural area (odds ratio [OR], 1.063; $P>.05$ ). Surprisingly, after controlling for size, the odds of colocation are actually greater for physicians in a frontier area than their counterparts in urban areas (OR, 1.289; $P<.01)$.

The final model in Table 1 includes interaction terms between the number of PCPs at location and level of rurality. These results indicate a size effect slightly lower in large rural areas compared with urban areas (OR, 0.907; 95\% confidence interval, 0.871-0.944), but the reverse is true for PCPs in the more rural areas, such as isolated rural settings (OR, 1.214; 95\% confidence interval, 1.0661.381). This relationship is displayed in Figure 3, which shows how the likelihood of colocation varies by site size for physicians in urban areas, large rural areas, and more rural locations (combining small rural, isolated rural, and frontier). In isolated and frontier rural areas, physicians in locations with few physicians are less likely to be colocated than urban PCPs. However, the likelihood of colocation rises sharply with size, reaching $100 \%$ for sites with 25 to 30 physicians. By contrast, the likelihood of colocation increases more gradually and nears 100\% only in locations with more than 100 to 110 PCPs.

\section{Limitations}

The data we use is silent about integration and collaboration among PCPs and behavioral health

Figure 2. Percentage of primary care physicians collocated with behavioral health providers by level of rurality and match precision.

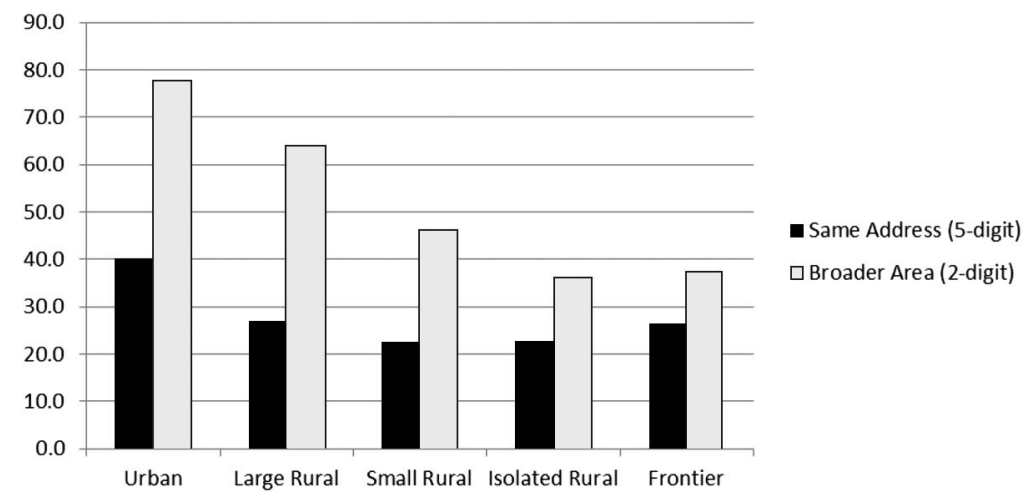


Table 1. Summary of Logistic Regression Analysis Examining Joint Impact of Size and Rurality on Colocation of Primary Care Physicians with Behavioral Health Providers

\begin{tabular}{lccc}
\hline Size and Location & Rurality Only & Rurality and Size & Rurality-Size Interaction \\
\hline Urban & Reference & Reference & Reference \\
Large rural & $0.552(0.533-0.571)^{*}$ & $0.758(0.730-0.787)^{*}$ & $0.921(0.843-1.006)$ \\
Small rural & $0.430(0.410-0.452)^{*}$ & $0.790(0.750-0.832)^{*}$ & $0.589(0.515-0.672)^{*}$ \\
Isolated rural & $0.439(0.400-0.482)^{*}$ & $1.063(0.961-1.174)$ & $0.807(0.652-0.999)^{\dagger}$ \\
Frontier & $0.537(0.489-0.590)^{*}$ & $1.289(1.166-1.425)^{*}$ & $1.118(0.899-1.391)$ \\
ln(size) & & $2.676(2.650-2.703)^{*}$ & $2.678(2.650-2.706)^{*}$ \\
Large rural*ln$(\operatorname{size})$ & & $0.907(0.871-0.944)^{*}$ \\
Small rural*ln$(\operatorname{size})$ & & & $1.185(1.105-1.269)^{*}$ \\
Isolated rural* $\ln ($ size $)$ & & & $1.214(1.066-1.381)^{*}$ \\
Frontier rural* $\ln ($ size $)$ & & & $1.106(0.966-1.265)$ \\
\hline
\end{tabular}

Data are odds ratios (95\% confidence intervals). Data are from the National Plan and Provider Enumeration System 2010 and the American Medical Association Masterfile 2010. The analysis is based on 207,955 primary care physicians in direct patient care. $\mathrm{Ln}$ (size) is the natural $\log$ of the number of primary care physicians located at the same location (street address). Colocation of primary care physicians and behavioral health providers also is based on the same, more precise location. The outcome variable for the three models is colocation of primary care physician with behavioral health providers.

*Significant at $1 \%$.

${ }^{\dagger}$ Significant at $5 \%$.

providers. Although PCPs may share an address with behavioral health providers, they may have no interaction with each other. Conversely, a rural physician may work closely with a psychologist a few towns over. The measure of colocation used in this article can serve as a valuable indicator of a potential relationship that could provide the basis for policies aimed at improving mental health care within primary care settings.

Another limitation of the data are that addresses analyzed and geocoded in both data sets are selfreported and contain inaccuracies. While the geo-

Figure 3. Association between colocation and primary care physicians (PCPs) at location.

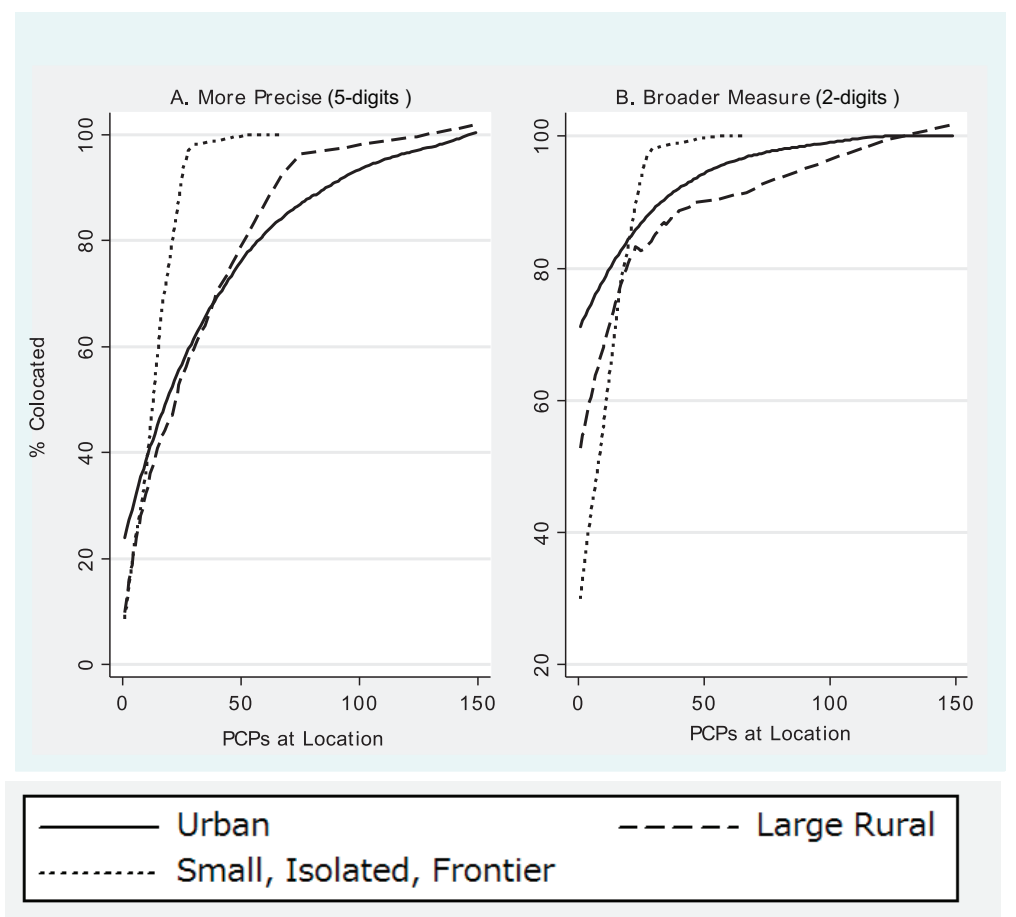


coding process strives to use the best possible address and apply the most accurate assignment of the longitude and latitude points, approximately $3 \%$ of addresses are geocoded at the 5-digit ZIP code. In addition, some health care providers may report their home or billing addresses rather than their practice address.

Since the NPI is required only for billing purposes, some providers may not be included in the NPPES. Furthermore, the NPPES database may overestimate the number of providers: all providers with an NPI are assumed to actively deliver clinical services, and providers who have retired have not necessarily been eliminated from the data. By combining NPPES data with AMA Masterfile data, we reduced the likelihood of overcounting physicians by including only those in direct patient care. Without a "master file" for behavioral health providers, we could not make a comparable correction for them.

\section{Discussion}

Successful expansion of integrated primary care delivery will require an array of strategies and interprovider arrangements, including in-house behavioral health staffing, coordination with proximal outside providers, and teleconsultation. Policymakers hoping to accelerate integration must first understand where the delivery of behavioral health and primary care services currently intersect and diverge. Absent this knowledge, policymakers will unlikely be able to recognize immediate opportunities for integration. Further, without a more complete understanding of where integration possibilities are, policymakers may not be able to ascertain where integration may simply need minimal incentives to occur and those geographical areas where greater policy and payment will need to be leveraged. Unfortunately, the current extent and distribution of primary care and mental health colocation and integration as it relates to provider density and practice proximity is unknown. In this article, we illuminated where behavior health and primary care proximity might offer a proxy for potential collaboration possibilities and where alternative strategies and support are likely needed to propel the integration of primary care and mental health forward to improve the health care delivery system for Americans.

The degree to which PCPs are colocated with behavioral health providers is positively associated with practice site size. This association explains some but not all the observed differences across level of rurality. While there are few large sites in more rural areas, findings show that they do exist, and they almost always contain a behavioral health provider. These findings suggest that support for integration in smaller practices is needed and adds to the growing call for facilitating change among smaller primary care practices. ${ }^{17}$ Current providers who have been trained as solo practitioners may need additional assistance and would benefit from training in the effective delivery of team-based care. Awareness of proximity informs opportunities for not only full integration of care but also coordination of care when integration is not possible or desired.

While some limitations of our colocation method to understand relationships between primary and behavioral health care providers are obvious, several are, nonetheless, worth noting. To understand national workforce patterns, we made opportunistic use of secondary and administrative data, which do not include information about actual employment, referral, or other relationships between providers nor evidence to understand where patient sharing is actually occurring. Our results can, therefore, only speak to the degrees to which potential relationships occur or could occur; for example, a part-time or even a full-time professional colocated within a primary care practice may be primarily employed at a mental health organization outside of the primary care practice building. Our results are best suited to demonstrating gaps in proximity, and our findings are consistent with well-documented shortages in rural areas of primary care mental health providers independent of one another. ${ }^{18-21}$ A plausible explanation of the low rates of colocation in rural areas is the absence of behavioral health providers. Recruiting and retaining these providers in rural areas is complicated because of economic, geographic, and sociocultural characteristics. ${ }^{21}$ Broadening the use of small-business loans, debt relief, enhanced infrastructure for telehealth opportunities, and additional payment incentives like those found to be beneficial in regional and international settings should all be considered among efforts to recruit and retain both PCPs and behavioral health providers in rural areas..$^{20,22,23}$ Incentive plans and innovative curricula also are needed to encourage behavioral health providers to train for the provision of services within a primary care environment. ${ }^{24-27}$ 


\section{Conclusions}

Despite evidence showing that integrating behavioral health care into primary care can improve patient outcomes ${ }^{28}$ and decrease health care costs, ${ }^{2}$ there remain many unanswered questions about who is integrating and where. ${ }^{29}$ Among these are payment policies separating physical and behavioral health care, workforce distribution, and supply deficiencies, particularly in rural areas.

The challenges inherent to delivering integrated primary care among physicians in smaller practices and in rural settings are many, as are the strategies required for their mitigation. To realize this potential, policies that both encourage and support integration are needed. Several key processes are needed to enable primary care providers to collaborate better with behavioral health providers: increased reimbursement and education at the practice level, ${ }^{7}$ workforce expansion, shared training, payment reform that favors team approaches, outcomes and population-based care to balance the isolating effects of fees for service, telehealth initiatives, and other incentives that support shared care. As we continue to better understand the importance of integration efforts, support can take place at the local community level as well as through state-based and national initiatives.

The United States must address the problem of fragmentation in health care delivery, and better integration of its health workforce through innovative delivery models is a critical first step. The inclusion of behavioral health providers into the largest platform of health care delivery, primary care, is an essential step toward the achievement of the nation's triple aim of decreasing overall health care cost, improving outcomes, and enhancing the patient experience. ${ }^{30}$ Understanding their current proximity and gaps in colocation is a critical first step-one that we hope galvanizes further research to understand how such proximity affects the actual team-based integrated care delivery and population health.

\section{References}

1. Hoffman C, Rice D, Sung HY. Persons with chronic conditions. Their prevalence and costs. JAMA 1996; 276:1473-9.

2. Simon GE, Katon WJ, VonKorff M, et al. Costeffectiveness of a collaborative care program for primary care patients with persistent depression. Am J Psychiatry 2001;158:1638-44.
3. Simon GE, VonKorff M, Barlow W. Health care costs of primary care patients with recognized depression. Arch Gen Psychiatry 1995;52:850-6.

4. deGruy F. Mental health care in the primary care setting. In: Donaldson MS, Yordy KD, Lohr KN, Vanselow NA, eds. Primary care: America's health in a new era. Washington, D.C.: Institute of Medicine; 1996:285-311.

5. Butler M, Kane RL, McAlpin D, et al. Integration of mental health/substance abuse and primary care. Evidence reports/technology assessments, no. 173 Rockville, MD: Agency for Healthcare Research and Quality; 2008. Available from: http://www.ncbi.nlm. nih.gov/books/NBK38632/. Accessed March 10, 2014.

6. Collins C, Hewson DL, Munger R, Wade T. Evolving models of behavioral health integration in primary care. May 2010. Available from: http://www. milbank.org/uploads/documents/10430EvolvingCare/ 10430EvolvingCare.html. Accessed March 10, 2014.

7. Robinson PJ, Reiter JT. Behavioral consultation and primary care: a guide to integrating services. New York: Springer; 2007.

8. Miller BF, Kessler R, Peek CJ, Kallenberg GA. A national agenda for research in collaborative care. Papers from the Collaborative Care Research Network Research Development Conference. Rockville, MD: Agency for Healthcare Research and Quality; 2011. Available from: http://www.ahrq.gov/research/ findings/final-reports/collaborativecare/collabcare.pdf. Accessed March 10, 2014.

9. Miller BF, Petterson S, Teevan Burke B, Phillips RL. Proximity of providers: colocating behavioral health and primary care and the prospects for an integrated workforce. Am Psychol. In press.

10. Peek CJ, National Integration Academy Council. Lexicon for behavioral health and primary care integration: concepts and definitions developed by expert consensus. Rockville, MD: Agency for Healthcare Research and Quality; 2013. Available from: http://integrationacademy.ahrq.gov/sites/default/files/ Lexicon.pdf. Accessed March 10, 2014.

11. Sieber WJ, Miller BF, Kessler RS, et al. Establishing the Collaborative Care Research Network (CCRN): a description of initial participating sites. Fam Syst Health 2012;30:210-23.

12. Frequently asked questions about the national provider identifier (NPI). Updated November 22, 2000. Available from: http://aspe.hhs.gov/admnsimp/faqnpi. htm. Accessed March 10, 2014.

13. WWAMI Rural Health Research Center. RUCA data. Travel distance and time, remote, isolated, and frontier. Available from: http://depts.washington.edu/ uwruca/ruca-travel-dist.php. Accessed December 8, 2013.

14. Petterson SM, Phillips RL, Bazemore AW, Koinis GT. Unequal distribution of the U.S. primary care workforce. Am Fam Physician 2013;87:Online. Available from http://www.graham-center.org/ 
online/graham/home/publications/onepagers/2013/ unequal-distr.html. Accessed March 10, 2014.

15. Petterson S, Burke M, Phillips R, Teevan B. Accounting for graduate medical education production of primary care physicians and general surgeons: timing of measurement matters. Acad Med 2011;86: 605-8.

16. Health Resources and Services Administration, Bureau of Health Professions. Projecting the Supply and Demand for Primary Care Practitioners through 2020. November 2013. Available from: http://bhpr. hrsa.gov/healthworkforce/supplydemand/usworkforce/ primarycare/. Accessed March 10, 2014.

17. Nutting PA, Crabtree BF, McDaniel RR. Small primary care practices face four hurdles-including a physician-centric mind-set-in becoming medical homes. Health Aff (Millwood) 2012;31:2417-22.

18. The number of practicing primary care physicians in the United States. Primary care workforce facts and stats no. 1. AHRQ publication no. 12-P001-2-EF. 2011; Rockville (MD): Agency for Healthcare Research and Quality; 2011. Available from: http:// www.ahrq.gov/research/pcwork1.htm. Accessed January 3, 2012.

19. American Psychological Association Center for Workforce Studies. How many practicing psychologists are there in the United States? Available from: http://www.apa.org/support/about/psych/numbersus.aspx\#answer. Accessed January 29, 2012.

20. Robiner WN. The mental health professions: workforce supply and demand, issues, and challenges. Clin Psychol Rev 2006;26:600-25.

21. Buykx P, Humphreys J, Wakerman J, Pashen D. Systematic review of effective retention incentives for health workers in rural and remote areas: towards evidence-based policy. Aust J Rural Health 2010;18: 102-9.

22. Ivey SL, Scheffler R, Zazzali JL. Supply dynamics of the mental health workforce: implications for health policy. Milbank Q 1998;76:25-58.

23. Australian Government, Department of Human Services. Medicare. Telehealth. Updated January 7, 2013. Available from: http://www.medicareaustralia.gov. au/provider/incentives/telehealth/. Accessed September 6, 2013.

24. The White House, Office of the Press Secretary. President announces new jobs initiatives for rural America. Press release, August 16, 2011. Available from: http://www.whitehouse.gov/the-press-office/ 2011/08/16/president-announces-new-jobs-initiativesrural-america. Accessed September 6, 2013.

25. Blount A, DeGirolamo S, Mariani K. Training the collaborative care practitioners of the future. Fam Syst Health 2006;24:111-9.

26. Blount FA, Miller BF. Addressing the workforce crisis in integrated primary care. J Clin Psychology Med Settings 2009;16:113-9.

27. Kessler R, Stafford D, Messier R. The problem of integrating behavioral health in the medical home and the questions it leads to. J Clin Psychol Med Settings 2009;16:4-12.

28. Unutzer J, Katon W, Callahan CM, et al. Collaborative care management of late-life depression in the primary care setting. JAMA 2002;288:2836-45.

29. Kathol RG, Butler M, McAlpine DD, Kane RL. Barriers to physical and mental condition integrated service delivery. Psychosom Med 2010;72:511-8.

30. Berwick DM, Nolan TW, Whittington J. The triple aim: care, health, and cost. Health Aff (Millwood) 2008;27:759-69. 